\title{
LA INCIDENCIA DISTRIBUTIVA DEL IMPUESTO A LAS GASOLINAS EN CHILE
}

\author{
Claudio A. Agostini \\ Universidad Adolfo Ibáñez \\ Johanna Jiménez \\ ILADES-Georgetown University
}

\begin{abstract}
Resumen: En este trabajo se analiza la incidencia distributiva del impuesto específico a las gasolinas en Chile, usando para ello la V y VI Encuestas de Presupuestos Familiares. La incidencia se calcula tanto respecto a la distribución de ingreso como de gasto, de tal forma de considerar las potenciales diferencias entre ingreso transitorio y permanente. Se estima el Índice de Suits como medida de la progresividad del impuesto y utilizando bootstrapping se calculan intervalos de confianza que permitan comparar estadísticamente cambios en la incidencia frente a cambios en el impuesto. Los resultados muestran que el impuesto es leve o moderadamente progresivo, con un grado de progresividad menor respecto al ingreso que al gasto. La simulación de una rebaja en la tasa
\end{abstract}

Claudio Agostini. Doctor en Economía, University of Michigan. Profesor Titular, Escuela de Gobierno, Universidad Adolfo Ibáñez, Santiago, Chile (agostini@uahurtado.cl).

JohanA JimÉnEz. Master of Arts in Economics, Georgetown University. Investigadora Asociada, ILADES-Georgetown University (johannajimenezb@ gmail.com). 
de impuestos de casi $42 \%$, como la implementada en 2008 , muestra que en términos de incidencia su efecto es reducir mínimamente la progresividad del impuesto.

Clasificación JEL: H22, C15, L9.

Palabras clave: incidencia tributaria, impuesto a los combustibles, índice de Suits.

Recibido: octubre 2011; aceptado: marzo 2012.

\section{THE DISTRIBUTIVE INCIDENCE OF GASOLINE TAX IN CHILE}

Abstract: This paper analyzes the distributive incidence of gasoline tax in Chile, using the Fifth and Sixth Family Budget Surveys. Incidence is calculated for income and expense distribution so that the potential differences between temporary and permanent income are taken into account. The Suits Index is estimated to measure the progressivity of the tax and confidence intervals are calculated by bootstrapping to make a statistical comparison between changes in incidence and changes in the tax. The results show that the tax is slightly or moderately progressive, but is less progressive in income than in expenses. The simulation of a reduction of nearly $42 \%$ in the tax rate, like the one implemented in 2008, reveals that the effect is, in terms of incidence, to reduce the progressivity of the tax minimally.

JEL Classification: H22, C15, L9.

Keywords: tax incidence, fuel tax, Suits index.

Received: October 2011; accepted: March 2012. 


\section{Introducción}

$\mathrm{C}_{1}$ consumo del petróleo y sus derivados está asociado a una serie de externalidades negativas. En el caso de las gasolinas, que son utilizadas principalmente por los automóviles, su consumo genera externalidades negativas en forma directa, como la contaminación (emisiones de dióxido de carbono, óxido de nitrógeno y monóxido de carbono), y también en forma indirecta, como la congestión y los accidentes de tránsito.

Por esta razón, un impuesto a las gasolinas es un impuesto óptimo que permite internalizar las externalidades y mejorar la asignación de recursos en la economía (Musgrave y Musgrave, 1989; Innes, 1996; Thorpe, 1997). En general, la evidencia empírica confirma los efectos teóricos del impuesto a las gasolinas en la reducción de externalidades negativas. Para Estados Unidos, por ejemplo, un impuesto de un dólar por galón reduce entre 15 y $20 \%$ el consumo de gasolinas, alrededor de $12 \%$ las millas manejadas y entre 16 y $18 \%$ los accidentes de tránsito (Haughton y Sarkar, 1996).

A pesar de sus beneficios en términos de eficiencia económica, el impuesto a las gasolinas es resistido y suele enfrentar desafíos importantes de economía política en su implementación (Goel y Nelson, 1999; Nivola y Crandall, 1995). En particular, cuando los precios de las gasolinas suben demasiado, la mayoría de las veces producto de aumentos en el precio del petróleo, se exige la rebaja o eliminación del impuesto. El caso de Chile no es distinto, pero se agrega un componente distributivo en la discusión pública basado en la percepción de que el impuesto es regresivo. Es así como entre los argumentos utilizados sistemáticamente por parlamentarios en el Congreso para exigir la eliminación del impuesto, se menciona recurrentemente su impacto negativo en la clase media ${ }^{1}$. Lamentablemente, no hay evidencia respecto a la

${ }^{1}$ Algunos ejemplos: "Este es un tema que no da para más, y que se hace insostenible al mantener un impuesto regresivo, que afecta a la clase media" (diputado Gustavo Hasbún, 26 de septiembre de 2011); "la solución no es revisar el actual sistema [...] sino rebajar temporalmente el impuesto específico en beneficio directo de una clase media que ya no puede seguir soportando las continuas alzas" (diputados Aldo Cornejo y Pablo Lorenzini, 26 de septiembre de 2011); "No hay duda que el impuesto específico a los combustibles está ahogando a la clase media" (senador Sergio Romero, 17 de enero de 2008); "una medida de este tipo [rebaja del impuesto] va en directo beneficio de la clase 
incidencia distributiva del impuesto a las gasolinas en Chile que permita confirmar o rechazar esta percepción y así poder evaluar los efectos distributivos que tendría la reducción o eliminación del impuesto.

El objetivo de este trabajo es precisamente analizar la incidencia distributiva del impuesto a las gasolinas y estimar el grado de regresividad o progresividad que tiene el impuesto. Utilizando los datos de consumo e ingreso de los hogares en las V y VI Encuestas de Presupuestos Familiares, se estima el Índice de Suits de progresividad tributaria. Posteriormente, a través de un bootstrapping, se generan intervalos de confianza que permiten evaluar el cambio en la progresividad/regresividad del impuesto a las gasolinas frente a cambios en la tasa del impuesto. Finalmente, se hace una simulación del impacto distributivo de la rebaja del impuesto a las gasolinas realizada en el año 2008.

Si bien el debate público y parlamentario se ha concentrado en el impuesto a las gasolinas, sería interesante y relevante estudiar también el impacto distributivo del impuesto al diésel. Sin embargo, este trabajo se limita a estimar la incidencia distributiva del impuesto a las gasolinas por dos razones. La primera es que el impuesto al diésel es pagado por quienes viajan en bus y por quienes utilizan automóviles con motor de diésel y no hay información disponible y representativa a nivel de hogares que permita realizar la estimación de incidencia. La segunda es que, dado que existe un crédito tributario del impuesto al diésel para el transporte público, el supuesto del $100 \%$ de traspaso del impuesto a precios finales, estándar en la literatura económica para el estudio de incidencia distributiva, no se cumple y se requiere una estimación de incidencia en precios por sector económico en toda la cadena de distribución, para lo cual tampoco hay datos disponibles. Por último, el impuesto a las gasolinas genera $80 \%$ de la recaudación de los impuestos a los combustibles, por lo que su relevancia es mayor en términos de incidencia tributaria.

media" (Senador Víctor Pérez, marzo de 2009); "Lo que está ocurriendo [alza en el precio de las gasolinas] está destruyendo el corazón de la clase media en Chile" (senador Juan Antonio Coloma, 11 de enero de 2008); "[El impuesto a los combustibles] tiene efectos negativos en un sector importante de los emprendedores chilenos, así como también en la clase media" (diputado Jaime Mulet, 29 de abril de 2009); "No es cierto que este impuesto afecte a la gente que tiene dinero, este impuesto afecta especialmente a los más pobres, este impuesto afecta a la gente de clase media" (senador Baldo Prokurica, 21 de junio de 2006). Declaraciones disponibles a través de las páginas web del Senado (www.senado.cl) y la Cámara de Diputados (www.diputados.cl). 
El resto del artículo continúa de la siguiente forma. En la sección 2 se describe el impuesto a las gasolinas en Chile. La sección 3 discute la evidencia existente en la literatura económica respecto a la incidencia distributiva y la forma de medirla. En la sección 4 se describen en detalle los datos que se utilizan para el análisis empírico. La sección 5 presenta y discute los resultados. En la sección 6 se muestra una simulación de los efectos de la rebaja tributaria de 2008. Finalmente, la sección 7 concluye y plantea aspectos relevantes para futuras investigaciones.

\section{El impuesto a las gasolinas en Chile}

En Chile el impuesto específico a los combustibles se estableció en 1986 (Ley 18.502) y no con el objetivo de reducir externalidades negativas sino que con el fin de financiar la recuperación del país tras el terremoto de 1985. Para las gasolinas automotrices se estableció un impuesto de tres unidades tributarias mensuales (UTM) ${ }^{2}$ por metro cúbico más el $70 \%$ de la diferencia entre US $\$ 233$ y el precio de venta sin impuestos de un metro cúbico. Para el diésel, el impuesto era $1,5 \mathrm{UTM} / \mathrm{m}^{3}$, más el 70\% de la diferencia entre USD196 y el valor de un metro cúbico del combustible. El componente variable del impuesto, sin embargo, fue posteriormente reducido en múltiples oportunidades hasta llegar a $0 \%$ en abril de 1988.

La Figura $\mathrm{N}^{\mathrm{o}} 1$ muestra la evolución del componente fijo del impuesto a las gasolinas automotrices y en el período 1986-2009.

Tal como se observa en la Figura $N^{\circ}$, la tasa de impuesto ha tenido varias modificaciones posteriores a la introducción del impuesto: se redujo a 2 UTM a fines de 1988, volvió a la tasa original de 3 UTM en enero de 1990, luego se aumentó a 3,4893 UTM en junio de 1991, a 4,4084 UTM en agosto de 1995, a 5,2 UTM en enero de 2000, y finalmente a 6 UTM en enero de 2001. Posteriormente el impuesto se disminuyó a 4,5 UTM en marzo de 2008 y a 3,5 UTM en septiembre de 2008, para luego volver a subir primero a 4 UTM en mayo de 2009 y después a 4,5 UTM en julio de 2009.

${ }^{2}$ Medida utilizada por el Servicio de Impuestos Internos para mantener los valores de los impuestos constantes en términos reales, por lo que se reajusta mensualmente por inflación. En octubre 2009: 1 UTM $=\$ 36.498$. 


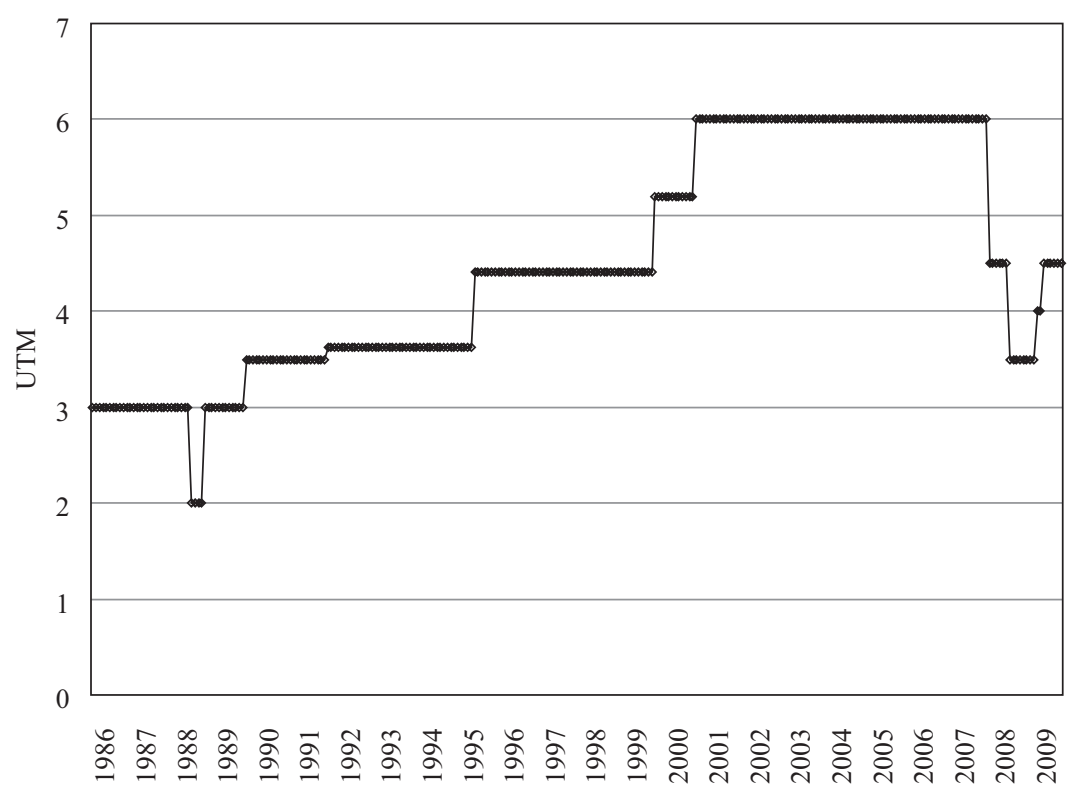

Adicionalmente al impuesto específico, las gasolinas también son gravados con IVA, que actualmente tiene una tasa de 19\%, el que se calcula sobre los precios sin tener en cuenta el impuesto específico ni el impuesto determinado por el Fondo de Estabilización de Precios de Combustibles derivados del petróleo (FEPC) ${ }^{3}$. El Cuadro $N^{\circ} 1$ muestra, para el 15 de octubre de 2009, la estructura del precio de venta de las gasolinas a mayoristas.

${ }^{3}$ El impuesto o subsidio definido por el FEPC se calcula a partir de la diferencia entre los precios de paridad y los precios de referencia. Los precios de paridad surgen de los precios internacionales del petróleo y los precios de referencia son calculados por el Ministerio de Minería a partir de la evaluación de los precios en períodos anteriores y las perspectivas futuras de los mismos (Ley 20.063 del 29 de Septiembre de 2005). Posteriormente, a partir de 2001 empezó a operar el SIPCO (Sistema de Protección al Contribuyente), que opera suavizando el impacto de las variaciones en los precios internacionales de los combustibles a través de un componente variable que se resta o se suma del componente base del impuesto específico a los combustibles (Ley $\mathrm{N}^{\circ} 20.493$ ). 


\begin{tabular}{lc}
\hline Ítem & Gasolinas \\
\hline Precio ENAP puesto en Concón & \\
Oleoducto Concón/Maipú & $55,4 \%$ \\
FEPC & $1,1 \%$ \\
Impuesto específico & $0,9 \%$ \\
IVA & $31,7 \%$ \\
Precio venta a mayoristas & $10,9 \%$ \\
\hline
\end{tabular}

* Promedio durante agosto 2009.

Finalmente, es importante mencionar que la recaudación del impuesto específico a las gasolinas representa alrededor del $0,8 \%$ del PIB. El año 2008, la recaudación de este impuesto generó el 3,1\% del total de ingresos tributarios, una disminución significativa respeto al año 2007 , cuando generó el 4,0\%, lo cual refleja los efectos de las dos rebajas tributarias de 2008. En promedio, la recaudación del impuesto a las gasolinas ha representado entre $72 \%$ y $80 \%$ de la recaudación total del impuesto a los combustibles, producto principalmente de la mayor tasa respecto al diésel.

\section{Evidencia y metodología de la incidencia distributiva}

Si bien los primeros análisis de incidencia tributaria se realizaron en el siglo XIX estudiando los cambios en la oferta y demanda de un bien producto de variaciones en los precios inducidas por impuestos, no es hasta el trabajo seminal de Harberger (1962) que el estudio de la incidencia de los impuestos adquirió mayor formalidad y relevancia en la literatura económica.

El análisis de Harberger (1962) se concentró en la incidencia tributaria que tiene el impuesto a las utilidades de las empresas en los factores de producción, pero la incidencia de un impuesto puede evaluarse en otras dimensiones igualmente relevantes dependiendo del tipo de impuesto: entre productores y consumidores de un bien o servicio, entre distintos consumidores según su ingreso o incluso entre consumidores o entre productores ubicados en distintas zonas geográficas. 
En el caso de la incidencia distributiva de un impuesto, el objetivo es determinar cómo cambia la fracción del ingreso destinada al pago de impuestos, es decir la carga tributaria, en la medida que aumenta el ingreso. Si esa fracción es creciente con el ingreso se considera que un impuesto es progresivo, si es proporcional se considera neutro y si cae al aumentar el ingreso se considera regresivo.

Una de las dificultades principales en el análisis de incidencia distributiva es cómo determinar la carga tributaria relevante, ya que puede haber discrepancias importantes entre la carga anual y la que soporta un consumidor a lo largo de su ciclo de vida. Por esta razón, el cálculo de incidencia distributiva a partir de datos de ingresos anuales puede ser una medida inexacta de la incidencia de un impuesto, en especial para impuestos al consumo (Poterba , 1989; Fullerton y Metcalf, 2002). Utilizar datos de gasto entonces, en vez de ingreso, puede ser una mejor alternativa, ya que ello reduce la importancia que tienen las variaciones anuales del ingreso e implícitamente incorpora en el análisis los patrones de ganancias, consumo y acumulación de activos.

Es así como Poterba (1989) muestra empíricamente que los impuestos a la gasolina, al tabaco y el alcohol en Estados Unidos son regresivos si se consideran los ingresos de las familias, pero el grado de regresividad disminuye considerablemente si se utilizan los gastos. Fullerton y Rogers (1991) comparan también la incidencia anual con la de ciclo de vida para los impuestos al ingreso y al consumo en Estados Unidos. Los resultados muestran que los impuestos al ingreso son menos progresivos y los al consumo menos regresivos en una perspectiva de ciclo de vida respecto a la incidencia anual. Sin embargo, muestran que para estimar la incidencia en el largo plazo (ciclo de vida) es relevante utilizar datos para períodos más largos, ya que los hogares cambian de decil de ingresos en el tiempo. Por ello, el análisis de incidencia de ciclo de vida es más bien complementario que sustituto del de incidencia anual. En forma similar, Metcalf (1994) estudia la incidencia distributiva del impuesto a las ventas en Estados Unidos. Sus resultados muestran que el impuesto es regresivo si se consideran los ingresos anuales de los hogares, pero es prácticamente proporcional si se analizan los gastos anuales de los mismos hogares.

En general, hay pocos estudios de incidencia distributiva en la literatura económica y éstos se concentran fuertemente en los impuestos al ingreso y a las ventas. Para el impuesto a las gasolinas existen sólo 
dos trabajos, ambos para Estados Unidos. El primero es el de Poterba (1991), que muestra que el impuesto a las gasolinas es regresivo, si bien la regresividad es mucho menor al considerar la distribución de gastos que la de ingresos de los hogares. En el trabajo se entrega mayor validez a los resultados utilizando gastos, ya que el consumo de los hogares no cambia sustancialmente durante el ciclo de vida y, por lo tanto, entrega mejor información sobre el ingreso de largo plazo. El segundo trabajo es el de Chernick y Reschovsky (1997), quienes hacen un cálculo de la incidencia del impuesto a las gasolinas a mediano plazo, en contraposición a las dos alternativas de incidencia anual o de ciclo de vida. Para ello, analizan la incidencia utilizando datos de ingreso y consumo promedio de gasolina de los hogares para un período de once años, con lo que se reduce el problema de cambio de deciles de los hogares. Los resultados muestran que el impuesto es regresivo, pero en menor grado respecto a la incidencia anual y a la de un período de cinco años.

La progresividad o regresividad de un impuesto puede simplemente mostrarse mediante la distribución de la carga tributaria por decil o quintil de ingreso, pero eso dificulta la comparación tanto entre distintos impuestos como para un mismo impuesto en el tiempo (antes y después de un cambio en la tasa o la base del impuesto por ejemplo). El índice propuesto por Suits (1977) para medir la progresividad de un impuesto elimina esta dificultad y sigue siendo el indicador más utilizado para estos efectos en la literatura económica tributaria.

Tal como se muestra en la Figura $N^{o}$ 2, el Índice de Suits se calcula a partir de una curva de Lorenz de la distribución acumulada de la recaudación del impuesto por decil respecto a la distribución acumulada de ingresos (curva OCB).

La intuición del Î́ndice de Suits es similar a la del coeficiente de Gini. Para un impuesto neutral la Curva de Lorenz sería idéntica a la diagonal $\mathrm{OB}$, por lo que la fracción de impuesto que paga cada contribuyente sería proporcional a su ingreso; para un impuesto progresivo la curva de Lorenz estaría bajo la diagonal $\mathrm{OB}$, por lo que la fracción de impuesto que paga cada contribuyente sería creciente con el ingreso; y finalmente, para un impuesto regresivo la curva de Lorenz estaría sobre la diagonal OB y la fracción de impuesto que paga un contribuyente sería decreciente respecto a su ingreso. De esta forma, el índice se define como:

$$
\mathrm{S}=1-(\triangle \mathrm{OABC} / \triangle \mathrm{OAB})=1-(\mathrm{L} / K)=1-(1 / 2) \int_{0}^{1} T(y) d y
$$


donde $\mathrm{L}$ es el área bajo la Curva de Lorenz y $\mathrm{K}$ es el área bajo la diagonal OB. Si el impuesto es proporcional las dos áreas son iguales y el índice es igual a cero; si el impuesto es progresivo el área bajo la Curva de Lorenz es menor que el área bajo la diagonal y el índice es positivo, con un valor máximo de 1; lo contrario ocurre con un impuesto regresivo y el índice toma valores negativos con un máximo de -1 . Una de las virtudes de este indicador es que cualquier cambio tributario que transfiera carga tributaria de un hogar o un individuo a otro hogar o individuo de mayor ingreso, aumenta el valor del índice. De igual forma cualquier transferencia de carga tributaria a un hogar de menor ingreso reduce el valor del índice. Adicionalmente, el índice de progresividad de un sistema tributario compuesto de múltiples impuestos corresponde simplemente a un promedio ponderado de los índices de cada uno de los impuestos por sí solo, donde los ponderadores son las tasas promedio de impuesto respectivas.

Una de las limitantes prácticas en el uso del Índice de Suits ha sido la dificultad de poder testear estadísticamente las diferencias entre dos índices o el cambio en el valor del índice frente a un cambio tributario. La dificultad radica principalmente en que las propiedades estadísticas del índice son desconocidas en muestras finitas. Sin embar-

\section{FIGURA N ${ }^{\circ}$ 2: $\quad$ ÍNDICE DE SUITS}

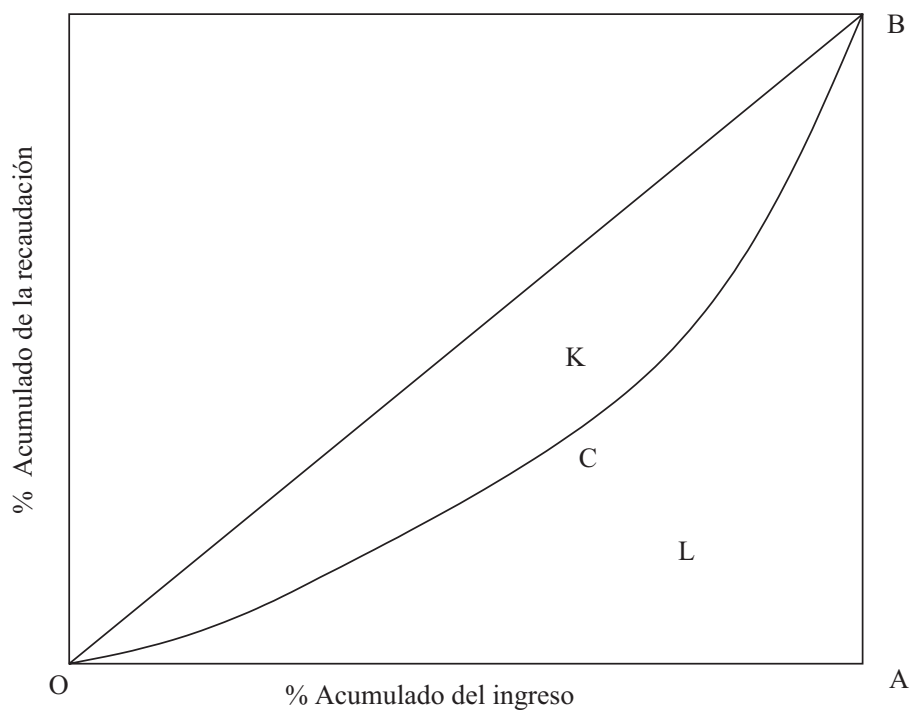


go, el trabajo de Anderson et al. (2003) plantea una forma de construir intervalos de confianza para el Índice de Suits utilizando bootstrapping, lo cual elimina dicha restricción.

\section{Datos}

En la estimación de la incidencia distributiva del impuesto a las gasolinas se utilizan los datos de la V y VI Encuestas de Presupuestos Familiares (EPF) que realiza cada diez años el Instituto Nacional de Estadísticas (INE). El objetivo de estas encuestas es determinar la estructura del gasto de consumo de los hogares y sus cambios en el tiempo, de tal forma de poder ajustar algunos indicadores, como la canasta del IPC o la línea de pobreza por ejemplo. Por esta razón y dada la periodicidad de la encuesta, cada diez años se mide el consumo final efectivo de los hogares, es decir, el gasto a precio contado de lo comprado por el hogar sin importar si el bien ya se encuentra pagado por completo o no. Esto último es particularmente relevante para medir el consumo de bienes durables. Adicionalmente, la encuesta registra el ingreso corriente disponible (ingreso líquido) de los hogares, el arriendo imputado ${ }^{4}$ en el caso de las viviendas propias y la existencia de deudas y créditos vigentes.

La V encuesta se realizó entre agosto de 1996 y julio de 1997, tiene una muestra de 8.455 hogares que son representativos de una población expandida de 5.233.796 y consideró sólo el Gran Santiago. La VI encuesta se efectuó entre noviembre de 2006 y octubre de 2007, consideró a 10.092 hogares que representan a una población de 9.433 .750 personas e incluyó no sólo al Gran Santiago sino también a las capitales regionales del país. En ambas encuestas se solicita a los hogares llevar un registro diario, durante quince días, de todos los gastos de todos los integrantes del hogar mayores de 15 años. Para los gastos periódicos se solicitó el último recibo en la $\mathrm{V}$ encuesta y los últimos recibos que correspondan a un mes de gasto en la VI encuesta ${ }^{5}$. En la VI encuesta se registran además las compras de bienes durables durante los últimos

${ }^{4}$ Se entiende por arriendo imputado el valor estimado que debería pagar el hogar si tuviera que arrendar la vivienda que posee.

${ }^{5}$ Gastos de arriendo, dividendo, agua, electricidad, gas a medidor, teléfono, TV cable, gastos comunes, contribuciones de bienes raíces, extracción de basura y servicios de educación. 
doce meses y los gastos en salud, educación y viajes de los últimos seis meses, a diferencia de $\mathrm{V}$ encuesta en que todos estos gastos aparecen registrados sólo para el mes anterior a la encuesta.

El Cuadro $\mathrm{N}^{\circ} 2$ muestra las estadísticas descriptivas básicas de los ingresos y los gastos de los hogares, en pesos de abril de 2007, para el Gran Santiago en las dos encuestas y para las capitales regionales y el total del país en la VI encuesta.

Los datos de la VI encuesta muestran un gasto promedio en los hogares menor que sus ingresos, lo cual podría reflejar un ingreso transitorio para 1996-1997 inferior al ingreso permanente. Lo contrario ocurre para 2006-2007 en que los gastos promedio por hogar son superiores a sus ingresos. Dada la discusión en la literatura respecto a las diferencias entre la incidencia distributiva de un impuesto anual y la de ciclo de vida, es relevante comparar las distribuciones de ingreso y gasto y posteriormente analizar la diferencia en la incidencia tributaria dependiendo de la distribución utilizada.

Las Figuras Nos. 3 y 4 muestran las distribuciones empíricas de ingreso y gasto de los hogares para la V y VI encuestas respectivamente. El test de Kolmogorov-Smirnov para comparar distribuciones no rechaza que la distribución de los ingresos sea igual a la distribución de los gastos en cada una de las encuestas. El test tampoco rechaza la igualdad de las dos distribuciones de ingreso, sin embargo se rechaza que la distribución de gastos de la $\mathrm{V}$ encuesta sea igual a la de la VI EPF. Este resultado parece contradictorio con la idea de que los gastos son más estables en el tiempo y reflejan mejor el ingreso permanente que los ingresos de un año, ya que la distribución del ingreso no ha cambiado estadísticamente entre 1996-1997 y 2006-2007 pero sí la de gastos. Sin embargo, las poblaciones de ambas encuestas son distintas

CUADRO N 2: $\quad$ ESTADÍSTICAS DESCRIPTIVAS DE INGRESO Y GASTO REAL

\begin{tabular}{|c|c|c|c|c|c|c|c|}
\hline \multirow[b]{2}{*}{ Área geográfica } & \multirow[b]{2}{*}{ EPF } & \multicolumn{3}{|c|}{ Ingreso real } & \multicolumn{3}{|c|}{ Gasto real } \\
\hline & & Hogares & Promedio & Desv. est. & Hogares & Promedio & Desv. est. \\
\hline \multirow[t]{2}{*}{ Gran Santiago } & $\mathrm{V}$ & 1.363 .622 & $\$ 665.871,5$ & $\$ 873.574,1$ & 1.363 .622 & $\$ 618.308,8$ & \$ 859.876,8 \\
\hline & VI & 1.632 .376 & $\$ 713.656,3$ & $\$ 910.468,1$ & 1.632 .376 & $\$ 740.738,7$ & \$ 922.436,7 \\
\hline Capitales regionales & $\mathrm{Vi}$ & 1.018 .457 & $\$ 571.212,4$ & $\$ 696.396,8$ & 1.018 .457 & $\$ 590.481,5$ & $\$ 687.529,6$ \\
\hline Nacional & VI & 2.650 .833 & $\$ 658.929,0$ & $\$ 837.611,5$ & 2.650 .833 & $\$ 683.009,5$ & $\$ 843.165,6$ \\
\hline
\end{tabular}


FIGURA N ${ }^{\circ}$ 3: $\quad$ DISTRIBUCIÓN DE INGRESO Y GASTO EN GRAN SANTIAGO 1996-1997

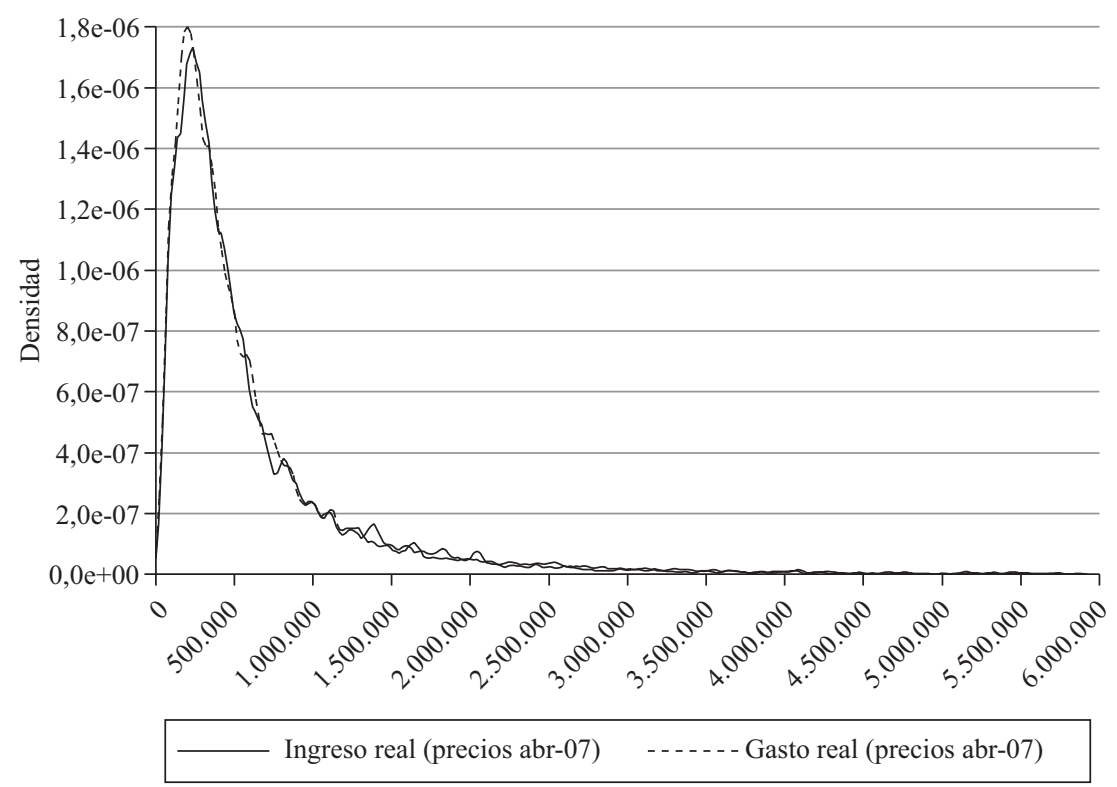

Fuente: Elaboración propia con datos de EPF 1996-1997. Ingresos y gastos en pesos de abril de 2007.

y la diferencia puede reflejar que la distribución de gastos en el Gran Santiago es distinta que la de las capitales regionales, si bien las distribuciones de ingresos no son distintas. De hecho, si se realiza el test para comparar la distribución de gastos entre ambas pero sólo para el Gran Santiago, no se rechaza que la distribución sea la misma.

A pesar de que las distribuciones de ingresos y gastos son estadísticamente iguales, la ubicación de cada hogar al interior de la distribución puede ser muy distinta dependiendo de si se usa su ingreso o su gasto, por lo que de igual forma puede ser relevante comparar la incidencia del impuesto a partir de los ingresos y los gastos de los hogares.

Si se hace el ejercicio de comparar la ubicación de cada hogar en los quintiles de ingreso y gasto, se observa que casi el 50\% de los hogares encuestados no presenta diferencia entre quintil de gasto e ingreso. Sin embargo, para el otro $50 \%$ de los hogares, tal como se observa en 


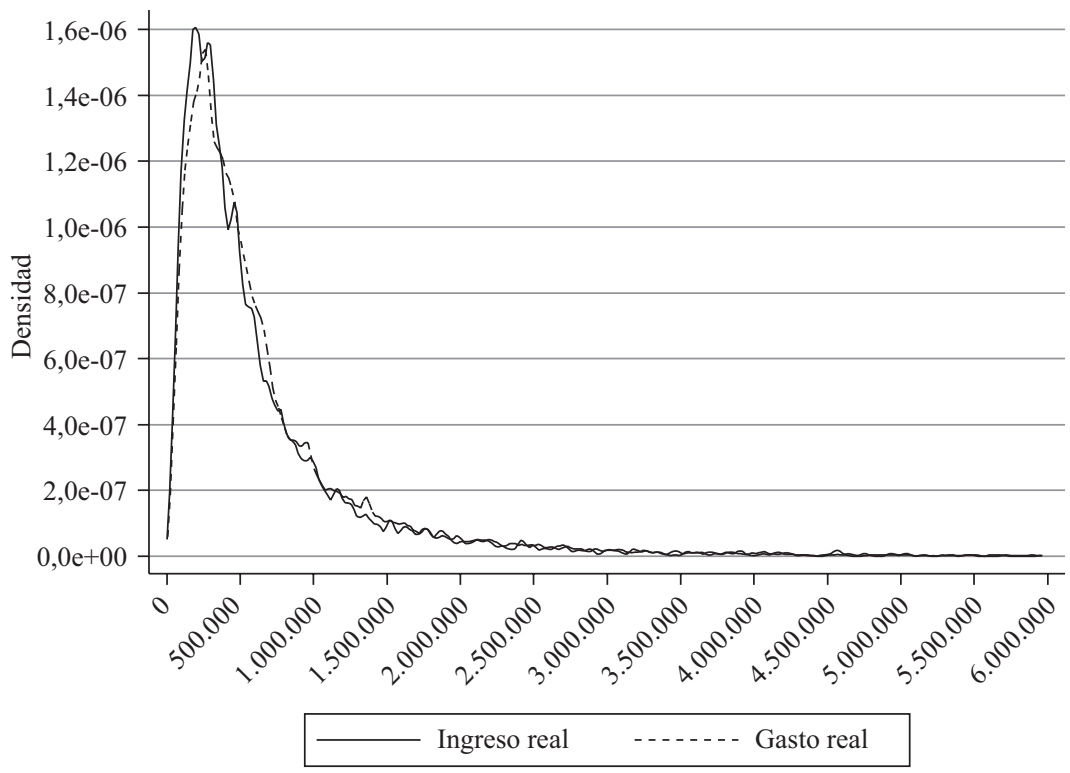

Fuente: Elaboración propia con datos de EPF 1996-1997. Ingresos y gastos en pesos de abril de 2007.

las Figuras Nos. 4 y 5, puede haber diferencias significativas de quintil. Es así como, por ejemplo, casi el 13\% de los hogares se encuentra dos o más quintiles más arriba o más abajo en la distribución de gasto respecto a la distribución de ingreso, lo cual es relevante para la interpretación posterior de los resultados de incidencia.

Si consideramos los gastos en automóvil, menos del 1\% de los hogares en el Gran Santiago registra la compra de un automóvil en 1996-1997, mientras que 7,2\% sí lo hace en 2006-2007. Si bien esta diferencia es consistente con el crecimiento del parque automotriz en la Región Metropolitana durante los últimos años, es posible que parte de la diferencia entre las dos encuestas se deba a que en la $\mathrm{V}$ encuesta se preguntó por el último mes mientras que en la VI se preguntó por los últimos doce meses. Sin embargo, para los efectos del impuesto a las gasolinas este cambio en la compra de vehículos podría tener efectos importantes en la incidencia distributiva del impuesto en el tiempo.

En forma similar, el 30\% de los hogares del Gran Santiago tenía algún nivel de gasto positivo en gasolinas en 1996-1997, mientras que 
FIGURA N ${ }^{\circ}$ 5: DIFERENCIA ENTRE QUINTILES DE GASTO E INGRESO DEL HOGAR 1996-1997

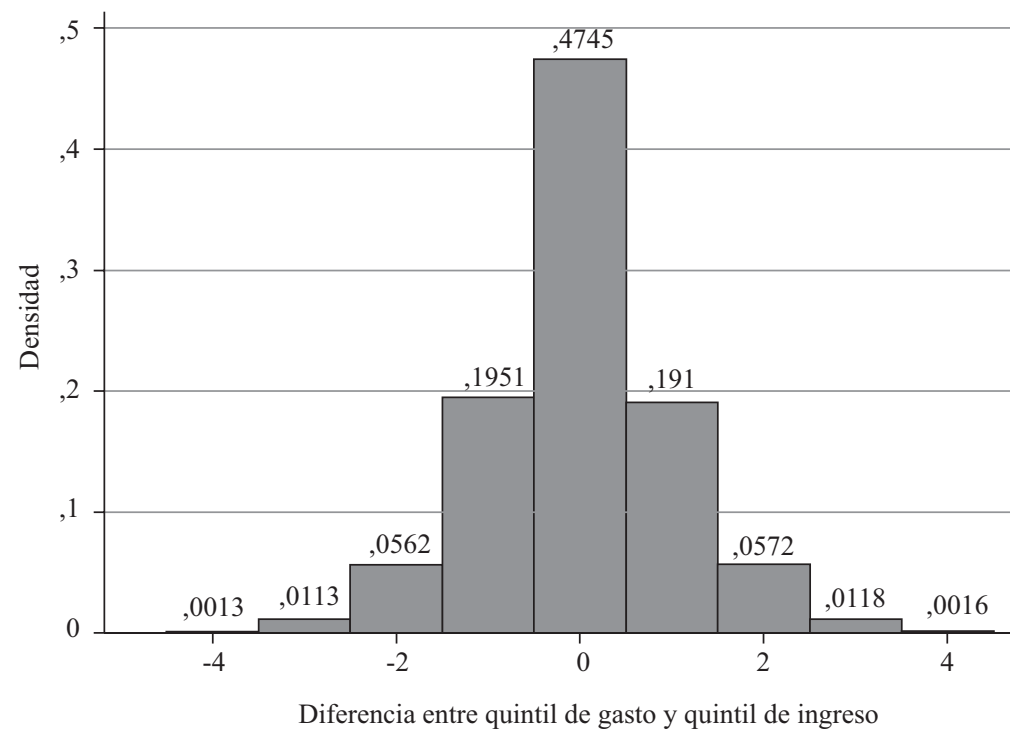

FIGURA N ${ }^{\circ}$ 6: $\quad$ DIFERENCIA ENTRE QUINTILES DE GASTO E INGRESO DEL HOGAR 2006-2007

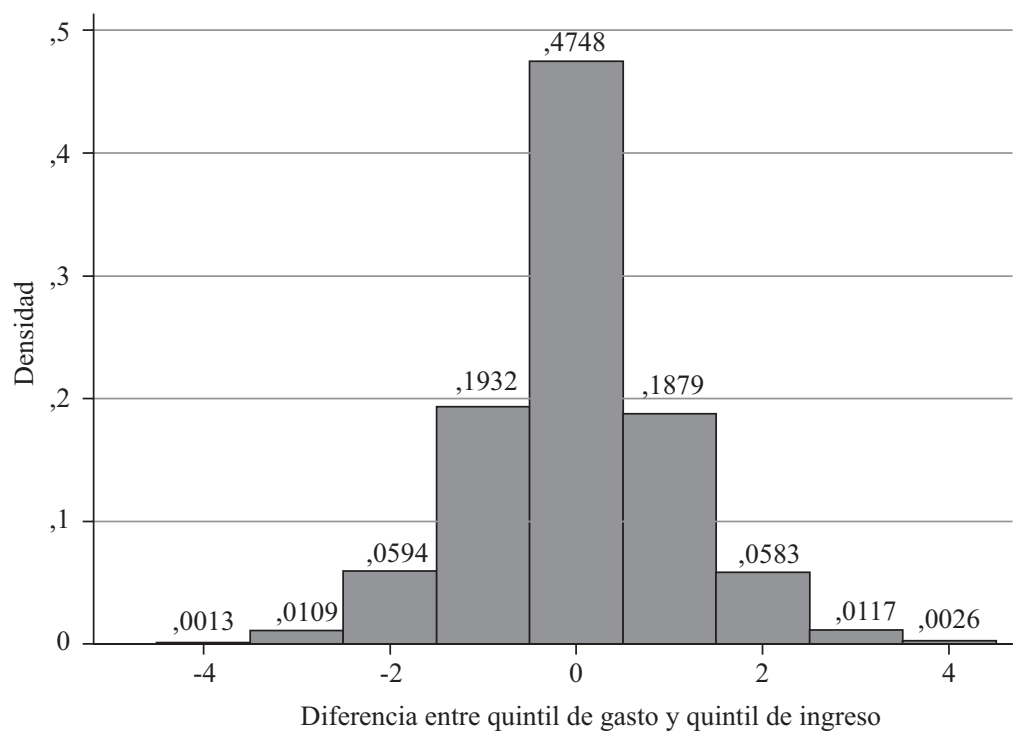


el 35,7\% lo tiene en 2006-2007. En las capitales regionales el 31,3\% gasta en gasolina un promedio de $\$ 53.484$, significativamente inferior al gasto promedio en Santiago de \$70.389. Las diferencias entre el Gran Santiago y las capitales regionales, tanto en la fracción de hogares que gastan en gasolina como el nivel de gasto, reflejan al mismo tiempo los distintos tamaños en el parque vehicular, el nivel de ingreso y el grado de urbanización de las ciudades. Por ello, es relevante en el análisis de incidencia estimar también por separado los efectos distributivos del impuesto en regiones y en el Gran Santiago.

El Cuadro $\mathrm{N}^{0} 3$ muestra cómo se distribuyen los hogares que tienen algún nivel de gasto en gasolina por decil de ingreso. La fracción de hogares que gasta en gasolina crece monotónicamente con el nivel de ingreso, lo cual es consistente con la distribución de automóviles por decil de ingreso que muestra la encuesta CASEN de 2006 (véase Gráfico $\mathrm{N}^{\mathrm{o}} 7$, infra $)^{6}$.

CUADRO No 3: $\quad$ FRACCIÓN DE HOGARES QUE GASTAN EN GASOLINA POR DECIL DE INGRESO

\begin{tabular}{cccr}
\hline & & \multicolumn{2}{c}{ VI EPF } \\
\cline { 3 - 3 } Decil de ingreso & V EPF & Gran Santiago & Nacional \\
\hline & & & \\
2 & $4,5 \%$ & $9,3 \%$ & $5,8 \%$ \\
3 & $5,7 \%$ & $16,9 \%$ & $10,3 \%$ \\
4 & $11,8 \%$ & $21,3 \%$ & $17,8 \%$ \\
5 & $14,9 \%$ & $22,1 \%$ & $22,6 \%$ \\
6 & $16,2 \%$ & $29,6 \%$ & $24,2 \%$ \\
7 & $22,5 \%$ & $38,5 \%$ & $31,1 \%$ \\
8 & $30,7 \%$ & $49,9 \%$ & $41,9 \%$ \\
9 & $47,1 \%$ & $63,6 \%$ & $49,9 \%$ \\
\hline
\end{tabular}

Ambas distribuciones, la de hogares que gastan en bencina y la de hogares que tienen automóvil, sugieren que el impuesto a las gasolinas podría ser progresivo ${ }^{7}$. Sin embargo, si los hogares de mayores

${ }^{6}$ El porcentaje de individuos que reportan tener automóvil en la encuesta CASEN de 2006 es de 27,3\%; de éstos, el 66\% son individuos de los quintiles $4^{\circ}$ y $5^{\circ}$.

${ }^{7}$ Las diferencias de fracción de gasto en gasolina entre deciles son estadísticamente significativas. 
FIGURA N N $^{\circ} \quad$ PORCENTAJE DE HOGARES CON AUTOMÓVIL POR DECIL DE INGRESO

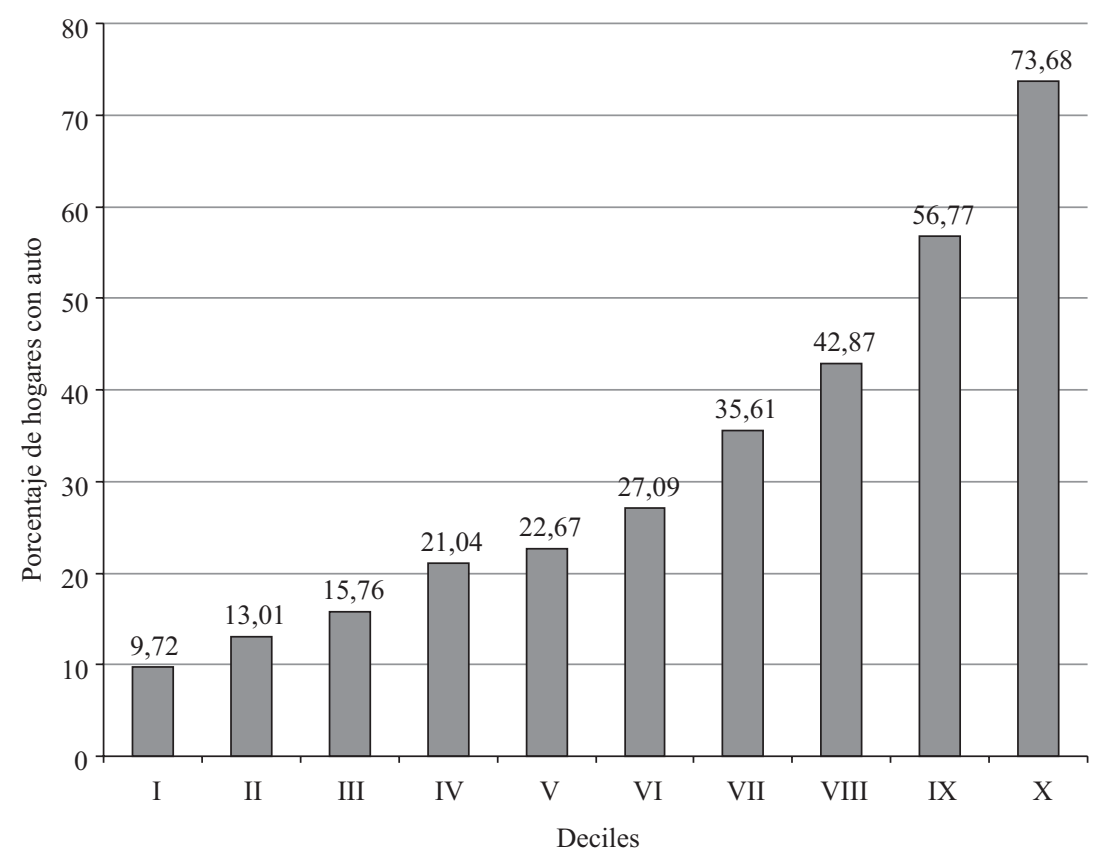

ingresos tienen automóviles más eficientes en el consumo de gasolina y/o manejan menos kilómetros al año, la incidencia podría ser menos progresiva o incluso neutral respecto a lo que estos datos sugieren. Por eso es relevante analizar en detalle el gasto en gasolina y calcular el Índice de Suits para medir la progresividad del impuesto, que es lo que se hace en la sección siguiente.

Un aspecto importante de destacar es que, durante el período transcurrido entre las dos encuestas, el número de hogares que gastan en gasolina en el Gran Santiago aumentó en todos los deciles, excepto en los dos de mayores ingresos. Una posible explicación es que los hogares de más altos ingresos tienen crecientemente automóviles a diésel, que tiene un impuesto menor que el de las gasolinas. En ese caso, el impuesto a las gasolinas debiera ser cada vez menos progresivo en el tiempo. 


\section{Resultados}

La estimación de la incidencia distributiva del impuesto a las gasolinas se realiza, siguiendo a Poterba (1991), respecto a los ingresos de los hogares y respecto a los gastos. Sin embargo, hay una consideración adicional que tomar en cuenta dado que en la encuesta se imputa un gasto por arriendo para los propietarios de vivienda. El valor del arriendo imputado es reportado por los mismos hogares y corresponde a su propia estimación de cuánto tendrían que pagar en arriendo por la vivienda que habitan, por lo que podría tener sesgos importantes que afectarían la estimación de incidencia si tienen alguna correlación con el nivel de ingreso o gasto (por ejemplo si los de más altos ingresos sobrestiman el arriendo respecto a los de menores ingresos o viceversa). Por ello, se calcula la incidencia respecto a los gastos con y sin la imputación de arriendo.

Un segundo elemento potencialmente relevante tiene que ver con el tamaño de los hogares. En promedio, los hogares de los deciles de menores ingresos tienen más miembros en el hogar que los de los deciles de más altos ingresos, por lo que la incidencia distributiva en los hogares puede ser distinta que la incidencia distributiva en las personas. En particular, dado que los hogares de los deciles más altos tienen una mayor proporción de automóviles, la incidencia del impuesto a la gasolina en los hogares puede ser menos progresiva que en los individuos. Para considerar esta posibilidad se calcula también la incidencia respecto a los ingresos y gastos per cápita en los hogares.

Por último, el gasto en automóviles en el período de la encuesta en que incurrieron algunos hogares podría sesgar la incidencia del impuesto, ya que sobrestima el gasto anual asociado al ingreso permanente para esos hogares, lo cual puede ocurrir en mayor proporción en algunos deciles específicos. Por esta razón se hacen dos análisis de sensibilidad, el primero restando el gasto en automóviles del gasto total de los hogares y el segundo eliminando de la muestra a los hogares que compraron automóvil.

A continuación se presentan los resultados de incidencia, primero para los hogares y luego per cápita, utilizando las distribuciones de ingresos, gastos, y gastos más la imputación de arriendo. En cada caso se calculó el Índice de Suits y, utilizando la metodología propuesta por Anderson, Gosh y Shoemaker (2003) se estimó un intervalo de con- 
fianza para el índice con un bootstrapping simultáneo de los vectores de ingresos, gastos, arriendos imputados y factores de expansión de los hogares.

\section{a. Incidencia en los hogares}

El Cuadro $\mathrm{N}^{\circ} 4$ muestra los resultados de incidencia tributaria en todo el país para la VI EPF. La fracción del gasto en gasolina varía entre $1,5 \%$ y $4,2 \%$ del ingreso de los hogares y entre $0,2 \%$ y $4,2 \%$ de los gastos. En general, la fracción del gasto crece en la medida que aumenta el ingreso pero más lentamente entre los primeros cinco deciles respecto a los últimos cinco deciles. El Índice de Suits muestra que el impuesto es progresivo tanto respecto al ingreso como al gasto, ya que en todos los casos, tal como muestra el intervalo de confianza, se rechaza la hipótesis de que el índice sea cero o menor que cero. Sin embargo, la progresividad en el caso del ingreso es muy baja y en términos de relevancia económica, más que estadística, no es muy distinta que la de un impuesto neutral. El impuesto es moderadamente progresivo respecto al gasto, y aun más respecto al gasto incluyendo el arriendo imputado, por lo que la incidencia del impuesto a las gasolinas en el largo plazo es más progresiva que la incidencia de un año en particular.

CUADRO N ${ }^{\circ}$ 4: $\quad$ INCIDENCIA DEL IMPUESTO A LA GASOLINA A NIVEL NACIONAL 2006-2007

\begin{tabular}{|c|c|c|c|c|c|c|}
\hline & \multicolumn{2}{|c|}{ Con ingreso total } & \multicolumn{2}{|c|}{ Con gasto total } & \multicolumn{2}{|c|}{$\begin{array}{l}\text { Con gasto total }+ \\
\text { arriendo imputado }\end{array}$} \\
\hline & Decil & Part. gast. gas. & Decil & Part. gast. gas. & Decil & Part. gast. gas. \\
\hline & 1 & $2,46 \%$ & 1 & $0,25 \%$ & 1 & $0,15 \%$ \\
\hline & 2 & $1,48 \%$ & 2 & $0,93 \%$ & 2 & $0,53 \%$ \\
\hline & 3 & $2,45 \%$ & 3 & $1,36 \%$ & 3 & $1,01 \%$ \\
\hline & 4 & $2,56 \%$ & 4 & $1,61 \%$ & 4 & $1,26 \%$ \\
\hline & 5 & $2,53 \%$ & 5 & $1,95 \%$ & 5 & $1,78 \%$ \\
\hline & 6 & $3,11 \%$ & 6 & $2,45 \%$ & 6 & $1,85 \%$ \\
\hline & 7 & $3,39 \%$ & 7 & $3,06 \%$ & 7 & $2,69 \%$ \\
\hline & 8 & $3,78 \%$ & 8 & $3,60 \%$ & 8 & $3,16 \%$ \\
\hline & 9 & $4,15 \%$ & 9 & $4,22 \%$ & 9 & $3,83 \%$ \\
\hline & 10 & $3,78 \%$ & 10 & $4,10 \%$ & 10 & $3,61 \%$ \\
\hline Índice de Suits & \multicolumn{2}{|r|}{0,05} & \multicolumn{2}{|r|}{0,14} & \multirow{3}{*}{\multicolumn{2}{|c|}{$\begin{array}{c}0,17 \\
{\left[\begin{array}{cc}0,150675 & 0,1888222\end{array}\right]} \\
0,00\end{array}$}} \\
\hline I.C. al $95 \%$, & {$[0,0294929$} & $90,0696405]$ & {$[0,11715$} & $38 \quad 0,1564493]$ & & \\
\hline $\mathrm{p}>\mathrm{z}$ & & 0,00 & & 0,00 & & \\
\hline
\end{tabular}


Los resultados para Gran Santiago obtenidos con los datos de las V y VI EPF se muestran en los Cuadros N ${ }^{\text {os. }} 5$ y 6 respectivamente. La fracción del gasto en gasolina en los hogares era bastante menor en 1996-1997 respecto a 2006-2007, lo cual refleja el aumento en la tasa de motorización en este período. A pesar de que la distribución de deciles no ha cambiado radicalmente, en ambos casos la fracción aumenta con el ingreso y lo hace más rápidamente en los últimos deciles que en los primeros; con la excepción del decil de más altos ingresos, la participación en el gasto para los dos deciles más bajos ha cambiado muy poco en estos diez años, aumentando entre 0,1 y 0,3 puntos porcentuales, mientras que para los dos deciles más altos aumentó alrededor de 1,5 puntos porcentuales.

El Índice de Suits muestra nuevamente que el impuesto es progresivo, levemente respecto al ingreso y moderadamente respecto al gasto que incluye arriendo imputado. El grado de progresividad en 1996-1997 es levemente inferior al existente en 2006-2007 respecto al gasto y levemente superior respecto al ingreso. Dado que el gasto refleja mejor el ingreso permanente y que la magnitud de los cambios es muy pequeña, la evidencia es que a pesar de que el nivel de ingreso en Chile ha aumentado significativamente en este período y el automóvil se ha hecho más accesible para muchos hogares, el impuesto no se ha hecho menos progresivo en el tiempo, al menos en el Gran Santiago. En el Apéndice 1 se muestran los resultados del análisis para las capitales regionales sin incluir Santiago. Los resultados son muy similares y no hay evidencia de que la progresividad del impuesto sea distinta en Santiago respecto al resto del país.

Un aspecto interesante de los resultados es que, si bien en general los deciles de más altos ingresos son los que soportan una mayor carga tributaria del impuesto a las gasolinas, es en el noveno decil y no en el décimo donde la incidencia del impuesto es mayor. Una explicación posible para esto, tal como se mencionó previamente, sería que los hogares del decil de más altos ingresos tienen automóviles a diésel. Alternativa o complementariamente, es posible que en el decil de ingresos más altos el jefe de hogar utilice un automóvil que es de propiedad de su empresa o que la empresa en la que trabaja incluya entre sus beneficios no monetarios los gastos de automóvil. En ambos casos, la incidencia del impuesto no recae directamente sobre el hogar. 
CUADRO N ${ }^{\circ}$ 5: $\quad$ INCIDENCIA DEL IMPUESTO A LA GASOLINA EN GRAN SANTIAGO 1996-1997

\begin{tabular}{|c|c|c|c|c|c|c|}
\hline & \multicolumn{2}{|c|}{ Con ingreso total } & \multicolumn{2}{|c|}{ Con gasto total } & \multicolumn{2}{|c|}{$\begin{array}{l}\text { Con gasto total }+ \\
\text { arriendo imputado }\end{array}$} \\
\hline & Decil & Part. gast. gas. & Decil & Part. gast. gas. & Decil & Part. gast. gas. \\
\hline & 1 & $1,38 \%$ & 1 & $0,09 \%$ & 1 & $0,09 \%$ \\
\hline & 2 & $0,90 \%$ & 2 & $0,62 \%$ & 2 & $0,40 \%$ \\
\hline & 3 & $1,39 \%$ & 3 & $0,89 \%$ & 3 & $0,53 \%$ \\
\hline & 4 & $1,59 \%$ & 4 & $1,42 \%$ & 4 & $0,71 \%$ \\
\hline & 5 & $1,53 \%$ & 5 & $1,75 \%$ & 5 & $1,44 \%$ \\
\hline & 6 & $1,84 \%$ & 6 & $2,45 \%$ & 6 & $1,60 \%$ \\
\hline & 7 & $2,21 \%$ & 7 & $2,69 \%$ & 7 & $2,16 \%$ \\
\hline & 8 & $2,82 \%$ & 8 & $3,17 \%$ & 8 & $2,53 \%$ \\
\hline & 9 & $3,04 \%$ & 9 & $3,31 \%$ & 9 & $2,80 \%$ \\
\hline & 10 & $2,49 \%$ & 10 & $2,93 \%$ & 10 & $2,57 \%$ \\
\hline Índice de Suits & \multicolumn{2}{|r|}{0,07} & \multicolumn{2}{|c|}{0,09} & \multicolumn{2}{|c|}{0,14} \\
\hline I.C. al 95\% & \multicolumn{2}{|c|}{$\left[\begin{array}{ll}0,0458967 & 0,09004\end{array}\right]$} & \multicolumn{2}{|c|}{$\left[\begin{array}{ll}0,0623857 & 0,111212\end{array}\right]$} & \multicolumn{2}{|c|}{$\left[\begin{array}{lll}0 ., 1168876 & 0,1635541\end{array}\right]$} \\
\hline $\mathrm{p}>\mathrm{z}$ & \multicolumn{2}{|c|}{0,00} & \multicolumn{2}{|c|}{0,00} & \multicolumn{2}{|c|}{0,00} \\
\hline
\end{tabular}

CUADRO N 6: $\quad$ INCIDENCIA DEL IMPUESTO A LA GASOLINA EN GRAN SANTIAGO 2006-2007

\begin{tabular}{|c|c|c|c|c|c|c|}
\hline & \multicolumn{2}{|c|}{ Con ingreso total } & \multicolumn{2}{|c|}{ Con gasto total } & \multicolumn{2}{|c|}{$\begin{array}{l}\text { Con gasto total + } \\
\text { arriendo imputado }\end{array}$} \\
\hline & Decil & Part. gast. gas. & Decil & Part. gast. gas. & Decil & Part. gast. gas. \\
\hline & 1 & $2,32 \%$ & 1 & $0,10 \%$ & 1 & $0,09 \%$ \\
\hline & 2 & $1,60 \%$ & 2 & $0,99 \%$ & 2 & $0,51 \%$ \\
\hline & 3 & $2,42 \%$ & 3 & $1,63 \%$ & 3 & $1,19 \%$ \\
\hline & 4 & $2,78 \%$ & 4 & $1,58 \%$ & 4 & $1,44 \%$ \\
\hline & 5 & $2,80 \%$ & 5 & $2,05 \%$ & 5 & $1,62 \%$ \\
\hline & 6 & $3,20 \%$ & 6 & $2,48 \%$ & 6 & $2,05 \%$ \\
\hline & 7 & $3,48 \%$ & 7 & $3,13 \%$ & 7 & $2,75 \%$ \\
\hline & 8 & $3,98 \%$ & 8 & $3,96 \%$ & 8 & $3,29 \%$ \\
\hline & 9 & $4,30 \%$ & 9 & $4,25 \%$ & 9 & $3,85 \%$ \\
\hline & 10 & $3,96 \%$ & 10 & $4,40 \%$ & 10 & $3,87 \%$ \\
\hline \multirow{3}{*}{$\begin{array}{l}\text { Índice de Suit } \\
\text { I.C. al } 95 \% \\
p>z\end{array}$} & \multicolumn{2}{|c|}{ s $\quad 0,05$} & \multicolumn{2}{|c|}{0,13} & \multirow{3}{*}{\multicolumn{2}{|c|}{$\begin{array}{c}0,16 \\
{[0,1390425 \quad 0,1853189]} \\
0,00\end{array}$}} \\
\hline & {$[0,020382$} & $80 ., 0697385]$ & {$[0,107503$} & 37 0.1551166] & & \\
\hline & & 0,00 & & 0,00 & & \\
\hline
\end{tabular}




\section{b. Incidencia per cápita}

En el Cuadro $\mathrm{N}^{\circ} 7$ se muestra la incidencia distributiva per cápita a nivel nacional a partir de la VI encuesta de 2006-2007. El Índice de Suits es levemente menor que para la incidencia en los hogares y vuelve a mostrar que el impuesto es leve o moderadamente progresivo. Sin embargo, en el caso de la incidencia en el ingreso no se rechaza estadísticamente que el impuesto pueda ser neutral.

CUADRO N ${ }^{\circ}$ 7: INCIDENCIA IMPUESTO A LA GASOLINA A NIVEL NACIONAL PER CÁPITA 2006-2007

\begin{tabular}{|c|c|c|c|c|c|c|}
\hline & \multicolumn{2}{|c|}{ Con ingreso total } & \multicolumn{2}{|c|}{ Con gasto total } & \multicolumn{2}{|c|}{$\begin{array}{l}\text { Con gasto total }+ \\
\text { arriendo imputado }\end{array}$} \\
\hline & Decil & Part. gast. gas. & Decil & Part. gast. gas. & Decil & Part. gast. gas. \\
\hline & 1 & $2,49 \%$ & 1 & $0,39 \%$ & 1 & $0,28 \%$ \\
\hline & 2 & $2,31 \%$ & 2 & $0,80 \%$ & 2 & $0,71 \%$ \\
\hline & 3 & $2,55 \%$ & 3 & $1,24 \%$ & 3 & $1,28 \%$ \\
\hline & 4 & $2,13 \%$ & 4 & $1,76 \%$ & 4 & $1,30 \%$ \\
\hline & 5 & $2,81 \%$ & 5 & $2,18 \%$ & 5 & $1,80 \%$ \\
\hline & 6 & $2,66 \%$ & 6 & $2,82 \%$ & 6 & $2,42 \%$ \\
\hline & 7 & $3,13 \%$ & 7 & $2,78 \%$ & 7 & $2,31 \%$ \\
\hline & 8 & $4,09 \%$ & 8 & $3,55 \%$ & 8 & $3,03 \%$ \\
\hline & 9 & $3,99 \%$ & 9 & $4,22 \%$ & 9 & $3,43 \%$ \\
\hline & 10 & $3,57 \%$ & 10 & $3,83 \%$ & 10 & $3,38 \%$ \\
\hline Índice de Suits & \multicolumn{2}{|c|}{0,02} & \multicolumn{2}{|r|}{0,11} & \multirow{3}{*}{\multicolumn{2}{|c|}{$\begin{array}{c}0,13 \\
{[0,11265480,153902]} \\
0,00\end{array}$}} \\
\hline I.C. al 95\% [ & {$[-0,007662$} & $270,0448986]$ & {$[0,08782$} & $03 \quad 0,1305416]$ & & \\
\hline $\mathrm{p}>\mathrm{z}$ & & 0,17 & & 0,00 & & \\
\hline
\end{tabular}

Los resultados para el Gran Santiago, que se presentan en los Cuadros Nos. 8 y 9, no son muy distintos a los de todo el país. La incidencia es práctica y estadísticamente neutral respecto al ingreso y progresiva respecto al gasto, incluyendo o no el arriendo imputado.

$\mathrm{Al}$ igual que en el caso de incidencia por hogares, no hay cambios significativos en la incidencia del impuesto entre 1996-1997 y 20072008, a pesar del aumento en el parque automotriz durante el período y el aumento en el ingreso promedio que hace accesible la compra de un automóvil a una mayor proporción de hogares. Para efectos de la discusión pública respecto al impuesto específico a la gasolina, éste es un re- 
CUADRO No 8: $\quad$ INCIDENCIA IMPUESTO A LA GASOLINA EN GRAN SANTIAGO PER CÁPITA 2006-2007

\begin{tabular}{|c|c|c|c|c|c|c|}
\hline & \multicolumn{2}{|c|}{ Con ingreso total } & \multicolumn{2}{|c|}{ Con gasto total } & \multicolumn{2}{|c|}{$\begin{array}{l}\text { Con gasto total }+ \\
\text { arriendo imputado }\end{array}$} \\
\hline & Decil & Part. gast. gas. & Decil & Part. gast. gas. & Decil & Part. gast. gas. \\
\hline & 1 & $2,55 \%$ & 1 & $0,41 \%$ & 1 & $0,41 \%$ \\
\hline & 2 & $2,34 \%$ & 2 & $0,80 \%$ & 2 & $0,80 \%$ \\
\hline & 3 & $2,07 \%$ & 3 & $0,95 \%$ & 3 & $0,95 \%$ \\
\hline & 4 & $2,58 \%$ & 4 & $1,15 \%$ & 4 & $1,15 \%$ \\
\hline & 5 & $2,23 \%$ & 5 & $2,23 \%$ & 5 & $2,23 \%$ \\
\hline & 6 & $2,42 \%$ & 6 & $2,38 \%$ & 6 & $2,38 \%$ \\
\hline & 7 & $3,03 \%$ & 7 & $3,40 \%$ & 7 & $3,40 \%$ \\
\hline & 8 & $3,98 \%$ & 8 & $3,14 \%$ & 8 & $3,14 \%$ \\
\hline & 9 & $3,70 \%$ & 9 & $3,90 \%$ & 9 & $3,90 \%$ \\
\hline & 10 & $3,05 \%$ & 10 & $3,43 \%$ & 10 & $3,43 \%$ \\
\hline Índice de Suits & \multicolumn{2}{|c|}{ s $\quad 0,01$} & \multicolumn{2}{|c|}{0,10} & \multicolumn{2}{|c|}{0,12} \\
\hline I.C. al 95\% [ & \multicolumn{2}{|c|}{$\left[\begin{array}{ll}-0,0612369 & 0,0494926\end{array}\right]$} & \multicolumn{2}{|c|}{$\left[\begin{array}{ll}0,0547405 & 0,1418431\end{array}\right]$} & \multicolumn{2}{|c|}{$\left[\begin{array}{ll}0,0828642 & 0,1669368\end{array}\right]$} \\
\hline $\mathrm{p}>\mathrm{z}$ & \multicolumn{2}{|c|}{0,84} & \multicolumn{2}{|c|}{0,00} & \multicolumn{2}{|c|}{0,00} \\
\hline
\end{tabular}

CUADRO N ${ }^{\circ}$ 9: $\quad$ INCIDENCIA IMPUESTO A LA GASOLINA EN GRAN SANTIAGO PER CÁPITA 1996-1997

\begin{tabular}{|c|c|c|c|c|c|c|}
\hline & \multicolumn{2}{|c|}{ Con ingreso total } & \multicolumn{2}{|c|}{ Con gasto total } & \multicolumn{2}{|c|}{$\begin{array}{l}\text { Con gasto total + } \\
\text { arriendo imputado }\end{array}$} \\
\hline & Decil & Part. gast. gas. & Decil & Part. gast. gas. & Decil & Part. gast. gas. \\
\hline & 1 & $2,26 \%$ & 1 & $0,42 \%$ & 1 & $0,34 \%$ \\
\hline & 2 & $2,52 \%$ & 2 & $0,82 \%$ & 2 & $0,96 \%$ \\
\hline & 3 & $2,72 \%$ & 3 & $1,69 \%$ & 3 & $1,27 \%$ \\
\hline & 4 & $2,06 \%$ & 4 & $1,80 \%$ & 4 & $1,24 \%$ \\
\hline & 5 & $2,79 \%$ & 5 & $2,26 \%$ & 5 & $2,03 \%$ \\
\hline & 6 & $2,95 \%$ & 6 & $2,79 \%$ & 6 & $2,27 \%$ \\
\hline & 7 & $3,51 \%$ & 7 & $2,57 \%$ & 7 & $2,40 \%$ \\
\hline & 8 & $4,16 \%$ & 8 & $3,88 \%$ & 8 & $3,26 \%$ \\
\hline & 9 & $4,21 \%$ & 9 & $4,28 \%$ & 9 & $3,49 \%$ \\
\hline & 10 & $3,65 \%$ & 10 & $4,11 \%$ & 10 & $3,51 \%$ \\
\hline Índice de Suits & \multicolumn{2}{|c|}{ s $\quad 0,02$} & \multicolumn{2}{|c|}{0,11} & \multirow{3}{*}{\multicolumn{2}{|c|}{$\begin{array}{c}0,13 \\
{[0,10726290,1547048]} \\
0,00\end{array}$}} \\
\hline I.C. al $95 \%$ & {$[-0,00501$} & $09,0480957]$ & {$[0,08241$} & 67 0,131297] & & \\
\hline $\mathrm{p}>\mathrm{z}$ & & 0,11 & & 0,00 & & \\
\hline
\end{tabular}


sultado relevante, ya que la evidencia no confirma la percepción de que el impuesto es regresivo ahora que el automóvil es un bien mucho más accesible para la mayoría de la población respecto a diez años atrás.

Finalmente, es importante señalar que, en general, estos resultados muestran que la incidencia distributiva per cápita no es muy distinta que la incidencia a nivel de hogares.

\section{c. Análisis de sensibilidad al gasto en automóviles}

Con el objeto de chequear la robustez de los resultados anteriores al potencial sesgo que podría introducir el gasto en automóviles efectuado por algunos hogares en los meses en que se realizaron las encuestas, se hicieron dos análisis de sensibilidad. Primero se estima la incidencia distributiva eliminando del gasto total el gasto en automóviles en que incurrieron los hogares. Segundo, se estima la incidencia eliminando las observaciones de hogares que compraron automóviles.

El Cuadro $\mathrm{N}^{\circ} 10$ muestra los resultados de incidencia a nivel nacional para eliminar el gasto en la compra de automóviles para la VI encuesta.

CUADRO N ${ }^{\circ}$ 10: INCIDENCIA A NIVEL NACIONAL SIN GASTO EN AUTOMÓVILES 2006-2007

\begin{tabular}{|c|c|c|c|c|c|c|}
\hline & \multicolumn{2}{|c|}{ Con ingreso total } & \multicolumn{2}{|c|}{ Con gasto total } & \multicolumn{2}{|c|}{$\begin{array}{l}\text { Con gasto total + } \\
\text { arriendo imputado }\end{array}$} \\
\hline & Decil & Part. gast. gas. & Decil & Part. gast. gas. & Decil & Part. gast. gas. \\
\hline & 1 & $2,46 \%$ & 1 & $0,36 \%$ & 1 & $0,27 \%$ \\
\hline & 2 & $1,48 \%$ & 2 & $1,00 \%$ & 2 & $0,55 \%$ \\
\hline & 3 & $2,45 \%$ & 3 & $1,60 \%$ & 3 & $1,10 \%$ \\
\hline & 4 & $2,56 \%$ & 4 & $1,77 \%$ & 4 & $1,55 \%$ \\
\hline & 5 & $2,53 \%$ & 5 & $2,19 \%$ & 5 & $1,89 \%$ \\
\hline & 6 & $3,11 \%$ & 6 & $2,59 \%$ & 6 & $1,96 \%$ \\
\hline & 7 & $3,39 \%$ & 7 & $3,35 \%$ & 7 & $3,04 \%$ \\
\hline & 8 & $3,78 \%$ & 8 & $3,77 \%$ & 8 & $3,32 \%$ \\
\hline & 9 & $4,15 \%$ & 9 & $4,44 \%$ & 9 & $3,79 \%$ \\
\hline & 10 & $3,78 \%$ & 10 & $4,55 \%$ & 10 & $4,05 \%$ \\
\hline Índice de Suits & \multicolumn{2}{|c|}{0,05} & \multicolumn{2}{|c|}{0,16} & \multicolumn{2}{|r|}{0,19} \\
\hline \multicolumn{7}{|c|}{ Int. Conf. I. } \\
\hline Suits al $95 \%$ & {$[0,029871$} & $0,0692606]$ & {$[0,138182$} & $270,1776695]$ & {$[0,1664$} & $890,205468]$ \\
\hline $\mathrm{p}>\mathrm{z}$ & & 0,00 & & 0,00 & & 0,00 \\
\hline
\end{tabular}


Tal como se aprecia en el cuadro, la progresividad del impuesto es un poco más alta, alrededor de 0,02 puntos en el Índice de Suits, que cuando se incluyen los gastos en automóvil. Esto se debe a que la proporción de hogares que compró automóviles en el período de la encuesta es mayor en los deciles de ingresos más altos. De hecho, en los primeros cuatro deciles casi no hay hogares que reporten haber comprado un automóvil. Adicionalmente, los automóviles comprados por los deciles mayores son de mayor valor, por lo que implican un mayor gasto. Como resultado, el gasto en gasolina aumenta en mayor proporción en los deciles de mayores ingresos cuando se eliminan los gastos en compra de automóviles y el impuesto se hace más progresivo. Los resultados para el Gran Santiago y para las capitales regionales son similares, la estimación punto muestra una progresividad un poco mayor del impuesto pero no hay cambios significativos ${ }^{8}$.

En el Apéndice 2 se encuentran los resultados del segundo análisis de sensibilidad que elimina a los hogares que compraron automóvil. Los resultados, si bien no son muy distintos, muestran un pequeño aumento en la progresividad del impuesto entre 0,03 y 0,05 puntos en el Índice de Suits respecto al análisis que incluye el gasto en automóviles de los hogares.

\section{Efectos de una rebaja en la tasa del impuesto a las gasolinas}

En 2008, con el objeto de reducir el impacto del alza en el precio del petróleo en los consumidores, se rebajó en forma transitoria el impuesto específico a las gasolinas de $6 \mathrm{UTM} / \mathrm{m}^{3}$ a $4,5 \mathrm{UTM} / \mathrm{m}^{3}$ hasta mayo de 2010. Para estimar el impacto de esta rebaja en la incidencia distributiva del impuesto se requiere información respecto a dos parámetros relevantes: la tasa de traspaso del impuesto a precios y de las elasticidades de precio de la demanda de los hogares. Lamentablemente, no hay estimaciones para ninguno de los dos en el caso de Chile. Sin embargo, es posible estimar una simulación del impacto utilizando algunos supuestos y la evidencia disponible para otros países. Esto permite al menos tener un rango de los efectos de la rebaja tributaria en la incidencia distributiva del impuesto.

${ }^{8}$ Los resultados para Santiago y las capitales regionales, dado que son similares, no se presentan por razones de espacio, pero están disponibles contactando a los autores. 
La teoría económica muestra que la tasa de traspaso de un impuesto a precios es de $100 \%$ en mercados competitivos con costos marginales constantes. Incluso si los costos marginales de corto plazo son crecientes, la tasa de traspaso en el largo plazo cuando todos los insumos son variables es de $100 \%$. Por otro lado, si la demanda es completamente inelástica, la tasa de traspaso es $100 \%$ independiente de la forma de la curva de oferta. En el caso de mercados no competitivos, la tasa de traspaso puede ser mayor o menor que 100\% incluso en el largo plazo (Katz y Rosen, 1985). El mercado de distribución minorista de combustibles suele considerarse un mercado competitivo, más allá de la existencia de algún grado de poder de mercado producto de la competencia espacial entre estaciones de servicio (Anderson et al., 2001), donde además la demanda es bastante inelástica. Por estas razones, en el análisis de incidencia distributiva, los estudios para Estados Unidos han asumido una tasa de traspaso a precios de 100\% (Poterba, 1991; Chernick y Reschovsky, 1997). De igual forma, en la estimación de los efectos de la rebaja tributaria en la incidencia distributiva del impuesto en Chile se asume una tasa de traspaso de $100 \%{ }^{9}$. Para el cálculo de precios posterior a la disminución del impuesto se utilizaron los precios mensuales promedio de la encuesta en todas las capitales regionales del país que realiza el Servicio Nacional del Consumidor (SERNAC).

El segundo componente relevante para la estimación tiene relación con la respuesta de los consumidores frente al cambio en el precio. Como se mencionó previamente, no hay estimaciones de elasticidad precio de la demanda de combustibles para Chile. Por esta razón se realizan dos estimaciones del impacto de la rebaja tributaria que intentan capturar un rango del efecto de acuerdo al cambio en el consumo de los hogares producto de la rebaja tributaria. La primera estimación considera que la demanda es completamente inelástica, por lo que los hogares siguen consumiendo la misma cantidad de gasolina anterior a la rebaja del impuesto. La segunda estimación utiliza la elasticidad de - 0,08 estimada por Hughes et al. (2008) para el período 2000-2006 en Estados Unidos, que es el período más cercano a la VI encuesta ${ }^{10}$.

${ }^{9}$ La evidencia para Chile es que la tasa de impuesto se traspasa en $100 \%$ en el mercado mayorista y en 140\% en el mercado minorista (Agostini, 2012).

${ }^{10} \mathrm{La}$ elasticidad precio estimada para Chile con datos anuales para el período 1960-1979 no es muy distinta, el estimador punto de la mejor especificación es $-0,1$ y el rango entre los distintos modelos estimados fluctúa entre $-0,01$ y $-0,16$ (Mujica, 1982). 
Los Cuadros N ${ }^{\text {os. }} 11$ y 12 muestran los resultados de la simulación con demanda inelástica y con elasticidad de $-0,08$ respectivamente para la incidencia en todo el país. Tal como se ve en los cuadros, el impacto de la rebaja es casi nulo respecto a la incidencia distributiva del impuesto. Los estimadores punto del Índice de Suits son marginalmente menores respecto a los resultados base para todo el país presentados en el Cuadro $\mathrm{N}^{\mathrm{o}} 4$, apuntando hacia una menor progresividad del impuesto, pero estadísticamente no hay cambios significativos ${ }^{11}$.

Sin duda que con elasticidades precio mayores el impacto podría aumentar y la rebaja haría que el impuesto fuera menos progresivo, pero no hay estimaciones que muestren que efectivamente la elasticidad precio de las gasolinas en Chile es mucho más alta y tampoco hay argumentos económicos que permitan suponer que la elasticidad en Chile es mayor que la estimada en la literatura económica para Estados Unidos. De hecho, la evidencia indirecta es que ante fluctuaciones importantes en el precio del petróleo el consumo agregado de gasolina no varía mayormente ${ }^{12}$.

Complementariamente, es posible también que la elasticidad precio de los deciles más bajos sea mayor que la de los deciles más altos, en cuyo caso la rebaja tributaria también podría potencialmente hacer menos progresivo el impuesto. Al igual que en el caso anterior, se requieren estimaciones de elasticidad por quintil o decil de ingreso que permitan realizar una simulación de este tipo en base a evidencia empírica para Chile. En la literatura económica existe en general muy poca evidencia respecto a elasticidades precio diferentes por quintil o decil de ingreso, una excepción es el trabajo de Wadud, Graham y Nolan (2005) para el caso de Estados Unidos. Con el objeto de al menos explorar el posible impacto de elasticidades distintas, utilizamos las elasticidades por quintil para Estados Unidos estimadas en dicho trabajo para simular el impacto de una rebaja en el impuesto a los combustibles en Chile (de 6 UTM/M $\mathrm{M}^{3}$ a 4,5 $\left.\mathrm{UTM} / \mathrm{M}^{3}\right)^{13}$. El Cuadro $\mathrm{N}^{\mathrm{o}} 13$ presenta los resultados de la estimación.

${ }^{11}$ Los resultados per cápita y para el Gran Santiago y las capitales regionales son muy parecidos y están disponibles contactando a los autores.

${ }^{12}$ Los datos de venta y precios de gasolinas para el período 1990-2008 publicados por la Comisión Nacional de Energía muestran una fluctuación promedio de $8,6 \%$ en el precio asociado a un cambio promedio de $0,4 \%$ en el consumo, lo cual implicaría una elasticidad precio de 0,56.

${ }^{13}$ Las elasticidades precio estimadas por Wadud et al. (2005) son de $-0,293$ para el quintil 5, de $-0,263$ para el quintil 4 , de $-0,203$ para el quintil 3 , de $-0,219$ para el quintil 2 y de $-0,351$ para el quintil 1 . 


\section{CUADRO N 11: SIMULACIÓN CON DEMANDA INELÁSTICA}

\begin{tabular}{|c|c|c|c|c|c|c|}
\hline & \multicolumn{2}{|c|}{ Con ingreso total } & \multicolumn{2}{|c|}{ Con gasto total } & \multicolumn{2}{|c|}{$\begin{array}{l}\text { Con gasto total }+ \\
\text { arriendo imputado }\end{array}$} \\
\hline & Decil & Part. gast. gas. & Decil & Part. gast. gas. & Decil & Part. gast. gas. \\
\hline & 1 & $2,26 \%$ & 1 & $0,23 \%$ & 1 & $0,14 \%$ \\
\hline & 2 & $1,37 \%$ & 2 & $0,90 \%$ & 2 & $0,51 \%$ \\
\hline & 3 & $2,25 \%$ & 3 & $1,28 \%$ & 3 & $0,97 \%$ \\
\hline & 4 & $2,36 \%$ & 4 & $1,50 \%$ & 4 & $1,16 \%$ \\
\hline & 5 & $2,33 \%$ & 5 & $1,86 \%$ & 5 & $1,68 \%$ \\
\hline & 6 & $2,86 \%$ & 6 & $2,30 \%$ & 6 & $1,69 \%$ \\
\hline & 7 & $3,11 \%$ & 7 & $2,79 \%$ & 7 & $2,53 \%$ \\
\hline & 8 & $3,48 \%$ & 8 & $3,31 \%$ & 8 & $2,88 \%$ \\
\hline & 9 & $3,82 \%$ & 9 & $3,88 \%$ & 9 & $3,52 \%$ \\
\hline & 10 & $3,47 \%$ & 10 & $3,79 \%$ & 10 & $3,33 \%$ \\
\hline \multirow{3}{*}{$\begin{array}{l}\text { Índice de Suits } \\
\text { I.C. al } 95 \% \\
\mathrm{p}>\mathrm{Z}\end{array}$} & \multicolumn{2}{|c|}{ s $\quad 0,05$} & \multicolumn{2}{|c|}{0,13} & \multirow{3}{*}{\multicolumn{2}{|c|}{$\begin{array}{c}0,16 \\
{[0,1486455 \quad 0,186518]} \\
0,00\end{array}$}} \\
\hline & {$[0,028076$} & {$[2 \quad 0,0709354]$} & {$[0,11465$} & $86 \quad 0,1539104]$ & & \\
\hline & & 0,00 & & 0,00 & & \\
\hline
\end{tabular}

CUADRO N ${ }^{\circ}$ 12: $\quad$ SIMULACIÓN CON DEMANDA CON ELASTICIDAD DE -0,08

\begin{tabular}{|c|c|c|c|c|c|c|}
\hline & \multicolumn{2}{|c|}{ Con ingreso total } & \multicolumn{2}{|c|}{ Con gasto total } & \multicolumn{2}{|c|}{$\begin{array}{l}\text { Con gasto total }+ \\
\text { arriendo imputado }\end{array}$} \\
\hline & Decil & Part. gast. gas. & Decil & Part. gast. gas. & Decil & Part. gast. gas. \\
\hline & 1 & $2,28 \%$ & 1 & $0,23 \%$ & 1 & $0,14 \%$ \\
\hline & 2 & $1,37 \%$ & 2 & $0,91 \%$ & 2 & $0,52 \%$ \\
\hline & 3 & $2,26 \%$ & 3 & $1,29 \%$ & 3 & $0,98 \%$ \\
\hline & 4 & $2,37 \%$ & 4 & $1,51 \%$ & 4 & $1,16 \%$ \\
\hline & 5 & $2,34 \%$ & 5 & $1,87 \%$ & 5 & $1,70 \%$ \\
\hline & 6 & $2,88 \%$ & 6 & $2,32 \%$ & 6 & $1,69 \%$ \\
\hline & 7 & $3,13 \%$ & 7 & $2,81 \%$ & 7 & $2,56 \%$ \\
\hline & 8 & $3,50 \%$ & 8 & $3,33 \%$ & 8 & $2,89 \%$ \\
\hline & 9 & $3,84 \%$ & 9 & $3,90 \%$ & 9 & $3,53 \%$ \\
\hline & 10 & $3,50 \%$ & 10 & $3,81 \%$ & 10 & $3,35 \%$ \\
\hline Índice de Suits & \multicolumn{2}{|r|}{0,05} & \multicolumn{2}{|r|}{0,13} & \multicolumn{2}{|r|}{0,16} \\
\hline I.C. al 95\% [ & \multicolumn{2}{|c|}{$\left[\begin{array}{ll}0,0292441 & 0,0697759\end{array}\right]$} & \multicolumn{2}{|c|}{$[0,11467860,1542601]$} & \multicolumn{2}{|c|}{$[0,1483120,1871742]$} \\
\hline $\mathrm{p}>\mathrm{z}$ & \multicolumn{2}{|c|}{0,00} & \multicolumn{2}{|c|}{0,00} & \multicolumn{2}{|c|}{0,00} \\
\hline
\end{tabular}


CUADRO N ${ }^{\circ}$ 13: $\quad$ SIMULACIÓN CON ELASTICIDAD DISTINTA POR QUINTIL (PARA EE.UU.)

\begin{tabular}{|c|c|c|c|c|c|c|}
\hline & \multicolumn{2}{|c|}{ Con ingreso total } & \multicolumn{2}{|c|}{ Con gasto total } & \multicolumn{2}{|c|}{$\begin{array}{l}\text { Con gasto total }+ \\
\text { arriendo imputado }\end{array}$} \\
\hline & Decil & Part. gast. gas. & Decil & Part. gast. gas. & Decil & Part. gast. gas. \\
\hline & 1 & $1,85 \%$ & 1 & $0,58 \%$ & 1 & $0,30 \%$ \\
\hline & 2 & $2,43 \%$ & 2 & $1,47 \%$ & 2 & $1,28 \%$ \\
\hline & 3 & $2,79 \%$ & 3 & $2,15 \%$ & 3 & $1,71 \%$ \\
\hline & 4 & $3,50 \%$ & 4 & $3,37 \%$ & 4 & $2,84 \%$ \\
\hline & 5 & $3,88 \%$ & 5 & $4,06 \%$ & 5 & $3,63 \%$ \\
\hline $\begin{array}{l}\text { Índice de Suits } \\
\text { Int. Conf. I. }\end{array}$ & 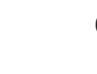 & 0,05 & & 0,13 & & 0,16 \\
\hline \multicolumn{3}{|c|}{$\begin{array}{l}\text { Int. Conf. I. } \\
\text { Suits al 95\% [0,0229392 } 0,0691381]\end{array}$} & \multicolumn{2}{|c|}{$[0,10669920,1538762]$} & \multicolumn{2}{|c|}{$\left[\begin{array}{ll}0,1387461 & 0,1841147\end{array}\right]$} \\
\hline $\mathrm{p}>\mathrm{z}$ & & 0,00 & & 0,00 & & 0,00 \\
\hline
\end{tabular}

Tal como se aprecia en el cuadro, si bien el Índice de Suits disminuye un poco respecto al caso en que la elasticidad se asume igual para todos los quintiles, el efecto sigue siendo marginal y el impuesto a las gasolinas sigue siendo un impuesto progresivo. No hay razones para pensar que los resultados serían muy distintos para Chile, incluso es más probable que las elasticidades sean mucho menores que en Estados Unidos, ya que la mayoría de los hogares no tiene automóvil. Por esta razón lo más probable es que el consumo total de gasolina en los quintiles de menores ingresos cambie muy poco producto de una disminución en el precio de la gasolina, ya que el consumo de la gran mayoría seguiría siendo cero $^{14}$.

Finalmente, es importante señalar que si bien las simulaciones realizadas para evaluar el efecto de la rebaja al impuesto a las gasolinas en su incidencia distributiva se basan en supuestos y estimaciones que pueden no cumplirse para el caso de Chile, al menos permiten iluminar el debate público al respecto. En particular, las simulaciones muestran que la rebaja tributaria no favorece particularmente a la clase media y

${ }^{14}$ Para tener una idea de órdenes de magnitud, se estimaron elasticidades por quintil utilizando la misma base de datos de la Encuesta de Presupuestos Familiares y los precios promedio mensuales que publica la Comisión Nacional de Energía. Si bien la estimación no considera variables instrumentales y otros ajustes econométricos que garantizarían resultados consistentes, las elasticidades estimadas fueron mucho menores que las de Estados Unidos. 
muy probablemente hace que el impuesto sea menos progresivo, favoreciendo en ese caso a los deciles de más altos ingresos que es lo contrario al objetivo buscado con la disminución del impuesto.

\section{Conclusiones}

El impuesto a las gasolinas aparece en el debate público cada vez que hay un shock en el precio internacional del petróleo que hace subir los precios en forma significativa. La visibilidad pública de los precios de las gasolinas se refuerza por el hecho de que ENAP anuncia semanalmente sus precios de venta a los distribuidores mayoristas. Como el impuesto a las gasolinas representa una fracción importante del precio, la presión pública para aliviar las alzas de precios se concentra en exigir una reducción del impuesto. En Chile, uno de los argumentos más fuertemente utilizados para presionar por una reducción o eliminación de dicho impuesto es que afecta mayormente a la clase media.

En este trabajo se estima la incidencia distributiva del impuesto a las gasolinas y los resultados muestran en forma robusta que la incidencia del impuesto es progresiva, levemente respecto al ingreso de los hogares y moderadamente respecto a los gastos. Los gastos de un hogar, en general, son más estables en el tiempo que los ingresos y por ello representan en mejor forma el ingreso permanente de los hogares. En ese sentido, los resultados muestran que el impuesto a las gasolinas es progresivo en el largo plazo cuando se considera el ingreso permanente de los hogares.

A partir de estos resultados no es posible afirmar que una reducción del impuesto a los combustibles beneficiaría en mayor proporción a la clase media, o al menos a los deciles que están alrededor de la media o la mediana de la distribución. Una simulación de la rebaja implementada en 2008 muestra que el efecto es casi nulo en la progresividad del impuesto y probablemente la disminuya, en cuyo caso beneficiaría en mayor proporción a los hogares de más altos ingresos.

Una estimación más precisa de los efectos de un cambio tributario requiere conocer cuánto del cambio de las tasas de impuestos se traspasa a precios finales de consumidores y cómo los consumidores cambian su consumo de gasolinas frente al cambio en el precio. Lamentablemente la evidencia empírica para Chile es escasa al respecto y se requieren futuras investigaciones que puedan proporcionarla. En particular, sería relevante estimar elasticidades precio de la demanda de gasolina por decil de ingreso (o de gasto), de tal forma de poder determinar con mayor precisión los efectos distributivos de un cambio en la tasa de impuestos. 
APÉNDICE 1:

INCIDENCIA EN LAS CAPITALES REGIONALES 2006-2007

\begin{tabular}{|c|c|c|c|c|c|c|}
\hline & \multicolumn{2}{|c|}{ Con ingreso total } & \multicolumn{2}{|c|}{ Con gasto total } & \multicolumn{2}{|c|}{$\begin{array}{l}\text { Con gasto total }+ \\
\text { arriendo imputado }\end{array}$} \\
\hline & Decil & Part. gast. gas. & Decil & Part. gast. gas. & Decil & Part. gast. gas. \\
\hline & 1 & $2,57 \%$ & 1 & $0,41 \%$ & 1 & $0,20 \%$ \\
\hline & 2 & $1,82 \%$ & 2 & $1,30 \%$ & 2 & $0,61 \%$ \\
\hline & 3 & $2,35 \%$ & 3 & $0,66 \%$ & 3 & $0,95 \%$ \\
\hline & 4 & $2,20 \%$ & 4 & $1,44 \%$ & 4 & $1,02 \%$ \\
\hline & 5 & $2,52 \%$ & 5 & $1,71 \%$ & 5 & $1,42 \%$ \\
\hline & 6 & $2,64 \%$ & 6 & $2,31 \%$ & 6 & $2,09 \%$ \\
\hline & 7 & $3,17 \%$ & 7 & $3,07 \%$ & 7 & $2,51 \%$ \\
\hline & 8 & $3,59 \%$ & 8 & $3,56 \%$ & 8 & $3,19 \%$ \\
\hline & 9 & $3,57 \%$ & 9 & $3,91 \%$ & 9 & $3,32 \%$ \\
\hline & 10 & $3,48 \%$ & 10 & $3,52 \%$ & 10 & $3,28 \%$ \\
\hline Índice de Suits & \multicolumn{2}{|r|}{0,04} & \multicolumn{2}{|c|}{0,13} & \multirow{2}{*}{\multicolumn{2}{|c|}{ 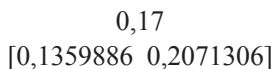 }} \\
\hline I.C. al 95\% & \multicolumn{2}{|c|}{$[-0,001431 \quad 0,0810516]$} & \multicolumn{2}{|c|}{$\left[\begin{array}{ll}0,0964477 & 0,1690369\end{array}\right]$} & & \\
\hline $\mathrm{p}>\mathrm{z}$ & \multicolumn{2}{|c|}{0,06} & \multicolumn{2}{|c|}{0,00} & \multicolumn{2}{|c|}{0,00} \\
\hline
\end{tabular}

APÉNDICE 2:

INCIDENCIA A NIVEL NACIONAL ELIMINANDO HOGARES QUE COMPRARON AUTOMÓVIL 2006-2007

\begin{tabular}{|c|c|c|c|c|c|c|}
\hline & \multicolumn{2}{|c|}{ Con ingreso total } & \multicolumn{2}{|c|}{ Con gasto total } & \multicolumn{2}{|c|}{$\begin{array}{l}\text { Con gasto total }+ \\
\text { arriendo imputado }\end{array}$} \\
\hline & Decil & Part. gast. gas. & Decil & Part. gast. gas. & Decil & Part. gast. gas. \\
\hline & 1 & $2,19 \%$ & 1 & $0,22 \%$ & 1 & $0,16 \%$ \\
\hline & 2 & $1,22 \%$ & 2 & $0,79 \%$ & 2 & $0,45 \%$ \\
\hline & 3 & $1,95 \%$ & 3 & $1,38 \%$ & 3 & $0,91 \%$ \\
\hline & 4 & $2,10 \%$ & 4 & $1,49 \%$ & 4 & $1,26 \%$ \\
\hline & 5 & $2,39 \%$ & 5 & $1,83 \%$ & 5 & $1,43 \%$ \\
\hline & 6 & $2,55 \%$ & 6 & $2,14 \%$ & 6 & $1,82 \%$ \\
\hline & 7 & $3,05 \%$ & 7 & $2,90 \%$ & 7 & $2,45 \%$ \\
\hline & 8 & $3,23 \%$ & 8 & $3,27 \%$ & 8 & $2,75 \%$ \\
\hline & 9 & $3,51 \%$ & 9 & $3,76 \%$ & 9 & $3,39 \%$ \\
\hline & 10 & $3,64 \%$ & 10 & $4,31 \%$ & 10 & $3,76 \%$ \\
\hline $\begin{array}{l}\text { Índice de Suits } \\
\text { Int. Conf. I. }\end{array}$ & \multicolumn{2}{|r|}{0,08} & \multicolumn{2}{|r|}{0,19} & \multicolumn{2}{|r|}{0,22} \\
\hline \multicolumn{3}{|c|}{$\begin{array}{l}\text { Int. Conf. I. } \\
\text { Suits al 95\% } \quad[0,0589805 \quad 0,1071837]\end{array}$} & \multicolumn{2}{|c|}{$\left[\begin{array}{lll}0,1652325 & 0,2101568\end{array}\right]$} & \multicolumn{2}{|c|}{$[0,19645090,2397536]$} \\
\hline $\mathrm{p}>\mathrm{z}$ & \multicolumn{2}{|c|}{0,00} & \multicolumn{2}{|c|}{0,00} & \multicolumn{2}{|c|}{0,00} \\
\hline
\end{tabular}




\section{REFERENCIAS}

Agostini, C. A. (2012), "Incidencia Tributaria en el Mercado de las Gasolinas en Chile”. Revista de Análisis Económico 27(2).

Anderson, John, Atrayee Ghosh y Paul Shoemaker (2003). "Confidence Intervals for the Suits Index", National Tax Journal 56(1).

Chernick, Howard y A. Reschovsky (1997). "Who Pays the Gasoline Tax?", National Tax Journal 50(2): 233-59.

Fullerton, Don y Gilbert E. Metcalf (2002). “Tax Incidence”, en Auerbach, Alan y Martin Feldstein (eds.), Handbook of Public Economics V.4, Elsevier Science.

Fullerton, Don y Diane Rogers (1991). "Lifetime versus Annual Perspectives on Tax Incidence", National Tax Journal 44(3): 277-287.

Goel, Rajeev y Michael A. Nelson (1999). "The Political Economy of Motor Fuel Taxation", Energy Journal 20(1).

Goodwin, P. B. (1992). "A Review of Fuel Demand Elasticities with Special Reference to Short and Long Run Effects of Price Changes", Journal of Transport Economics and Policy 25.

Harberger, Arnold (1962). "The Incidence of the Corporation Income Tax", Journal of Political Economy 70(3).

Haughton, Jonathan y Soumodip Sarkar (1996). "Gasoline Tax as a Corrective Tax: Estimates for the United States, 1970-1991”, The Energy Journal, International Association for Energy Economics, vol. 17(2): 103-126.

Hughes, Jonathan, Christopher Knittel y Daniel Sperling (2008). "Evidence of a Shift in the Short-Run Price Elasticity of Gasoline Demand", Energy Journal 29(1).

INE. Metodología, VI Encuesta de Presupuestos Familiares 2006-2007. Publicada por el Instituto Nacional de Estadísticas-INE en http://www. ine.cl.

Base de datos, V Encuesta de Presupuestos Familiares 19961997. Publicada por el Instituto Nacional de Estadísticas-INE en http://www.ine.cl.

Innes, Robert (1996). "Regulating Automobile Pollution Under Certainty, Competition, and Imperfect Information", Journal of Environmental Economics and Management 31 (September): 219-239.

Metcalf, Gilbert E. (1994). "Life Cycle versus Annual Perspectives on the Incidence of a Value Added Tax", Tax Policy and the Economy 8: 45-64.

Mujica, R. (1982). "Análisis de la Demanda y Predicción del Consumo de Hidrocarburos en Chile: 1980-2000”, Cuadernos de Economía 19(58).

Musgrave, R. A. y P. B. Musgrave (1989). Public Finance in theory and Practice, McGraw-Hill, New York.

Nivola, Pietro y Robert Grandall (1995). The Extra Mile, Brookings Institution, Washington DC.

Poterba, James (1989). "Lifetime Incidence and the Distributional Burden of Excise Taxes", American Economic Review 79(2): 325-330. 
(1991). "Is the Gasoline Tax Regressive?", Tax Policy and the Economy 5, NBER, MIT Press.

Suits, Donald (1977). "Measurement of Tax Progressivity", American Economic Review 67(4): 747-752.

Thorpe, Steven G. (1997). "Fuel Economy Standards, New Vehicle Sales and Average Fuel Efficiency", Journal of Regulatory Economics 11(3).

Wadud, Z., D. J. Graham y R. B. Noland (2005). "Modelling Fuel Demand for Different Socio-Economic Groups", Applied Energy 86: 2740-2749. 